\title{
Atypical Clinical and Neuroimaging Findings in Leber's Hereditary Optic Neuropathy: a Case Report
}

\author{
Alia Arianti ${ }^{1,2}$, Hideki Chuman ${ }^{1 *}$, Natsuki Kajihara ${ }^{1}$, Natsuko Sakamoto ${ }^{1}$ and Nobuhisa Nao-IM.D ${ }^{1}$ \\ ${ }^{1}$ Department of Ophthalmology, University of Miyazaki, Japan \\ ${ }^{2} J a k a r t a$ Eye Center, Indonesia
}

Submission: May 15, 2018; Published: May 31, 2018

*Corresponding author: Hideki Chuman, Department of Ophthalmology, Faculty of medicine, University of Miyazaki, 5200 Kihara Kiyotake Miyazaki city, Miyazaki Prefecture, Japan, Tel: 81-985-85-2806; Fax: 81-985-84-2065; Email: hchuman@med.miyazaki-u.ac.jp

\section{Abstract}

We report a case of Leber's hereditary optic neuropathy (LHON) with atypical clinical features and optic nerve abnormality defined by MRI, mimicking optic neuritis at initial presentation.

\section{Introduction}

Leber's hereditary optic neuropathy (LHON) is a maternally inherited bilateral optic neuropathy caused by mitochondrial DNA point mutation, predominantly in young male [1,2] The pathophysiology involves a primary point mtDNA mutations causing failure of the electron transport oxidative phosphorylation pathway, with the end result of selective retinal ganglion cells (RGC) apoptosis [1,3]. The most common type of mutation found in Japan is the mt11778 point mutation, accounting for $86.5 \%$ of all reported cases [3]. The presence of specific mitochondrial point mutation does not guarantee the development of symptomatic LHON later in individuals harboring the mutation. Incomplete penetrance is shown as only around $50 \%$ of males and $10 \%$ of females with the mutation will later develop visual loss [2,4-6].

Clinically, symptomatic phase of LHON is characterized by acute or subacute bilateral painless central visual loss with typical fundus findings [4,5]. Magnetic resonance imaging (MRI) findings are usually normal, with no apparent optic nerve or brain abnormalities [7]. However, reports of visual pathway hyperintensity and enhancement, particularly around the optic nerve and chiasm on MRI have been described, demonstrated an atypical findings of LHON [5,8-13]. Presence of atypical clinical findings in acute phase of LHON often create an overlapping features to other optic neuropathies, including optic neuritis, therefore creating additional challenges in its diagnosis $[14,15]$.

In our experience in Japan, presence of optic nerve abnormalities on MRI during acute phase of LHON is rare. We report a case of LHON with mt14484 mutation with atypical optic nerve hyperintensity and contrast enhancement on MRI which persist over to the atrophic phase, creating a challenge in the diagnosis of the disease. These findings might also suggest an inflammatory component of the optic nerve, arising new question of whether this is an atypical clinical feature of LHON, or a coincidental finding of LHON mutation in otherwise an optic neuritis attack, or whether optic neuritis triggered the symptomatic phase of LHON in this patient?

\section{Case Report}

A 42-year old man presented with sudden LE photophobia and metamorphopsia on October 2016. He also experienced numbness on four extremities. The patient was diagnosed with idiopathic optic neuritis and treated with 3 cycles of intravenous steroid before being referred to Miyazaki University Hospital (MUH) on February 2017. His past medical history was unremarkable, except for history of treated Hepatitis B infection 25 years ago and well-controlled dyslipidemia since one year. There was a history of smoking, but the patient has quit long before the symptom onset. There was a history of moderate daily alcohol consumption. No history of trauma, medication, or hormonal problem was present; and there was no family history of eye diseases.

On the initial symptom onset, the ophthalmological examination showed a swollen leftoptic disc (Figure 1). Fluorescein angiography examination showed a swollen optic disc with late-phase dye leakage, typical of optic neuritis on the LE (Figure 2). No other abnormalities were found on FA. Brain magnetic resonance imaging revealed a swollen, hyper intense left optic nerve on T2-weighted and diffusion weighted imaging (DWI) (Figure 3). No gadolinium contrast enhancement was 


\section{JOJ Ophthalmology}

performed at this moment. A total of 3 cycles of pulse IV steroid injections was given, and slight subjective improvement of vision was noted, and disappearance of the numbness. Work up for AQP4 antibody was negative. Spinal MRI was within normal limits, except for a minor cervical disc herniation, and no other supporting signs of NMO were found. Two months after the last steroid administration, the visual acuity remained stable with RE VA of 2.0 and LE 0.8. However, the left visual acuity worsen later on the same month and the patient was referred to Miyazaki University Hospital.
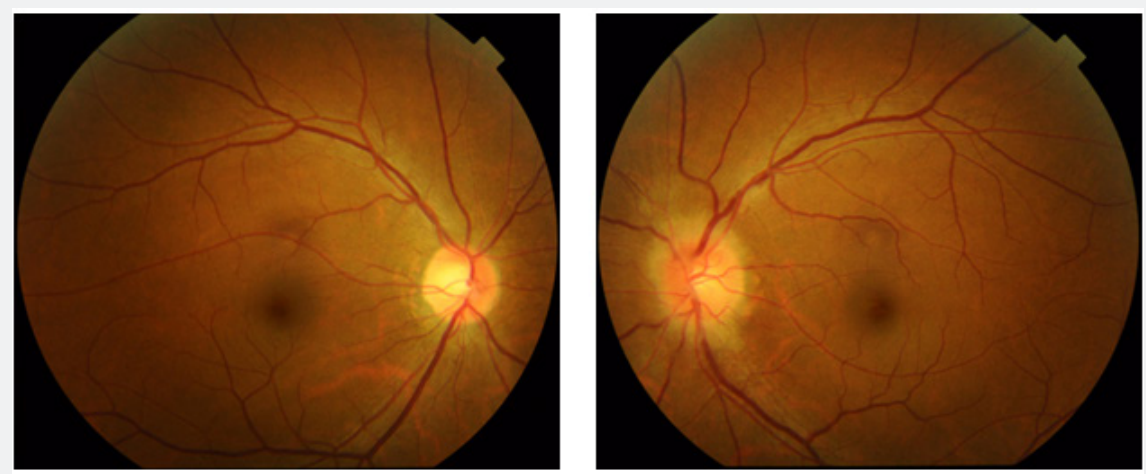

Figure 1: Swollen optic disc of the LE on initial presentation. Optic disc of the RE appears normal.
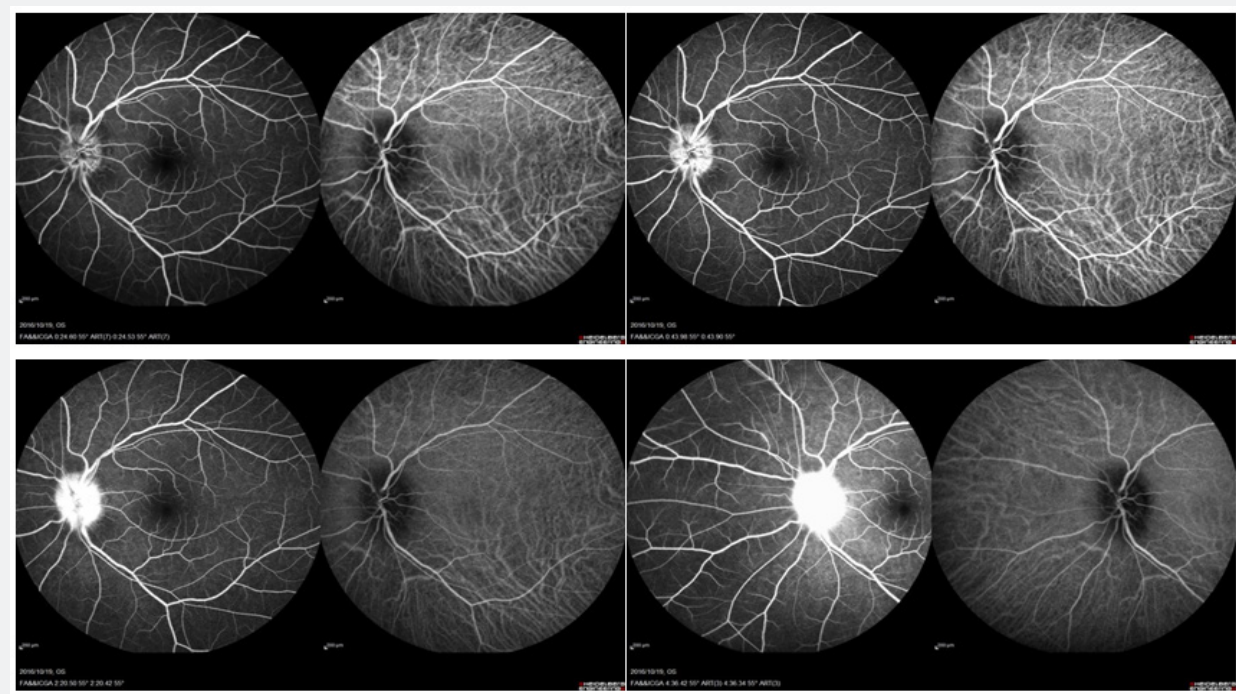

Figure 2: Initial fluorescein angiography showed swollen left optic disc with late-phase disc leakage.

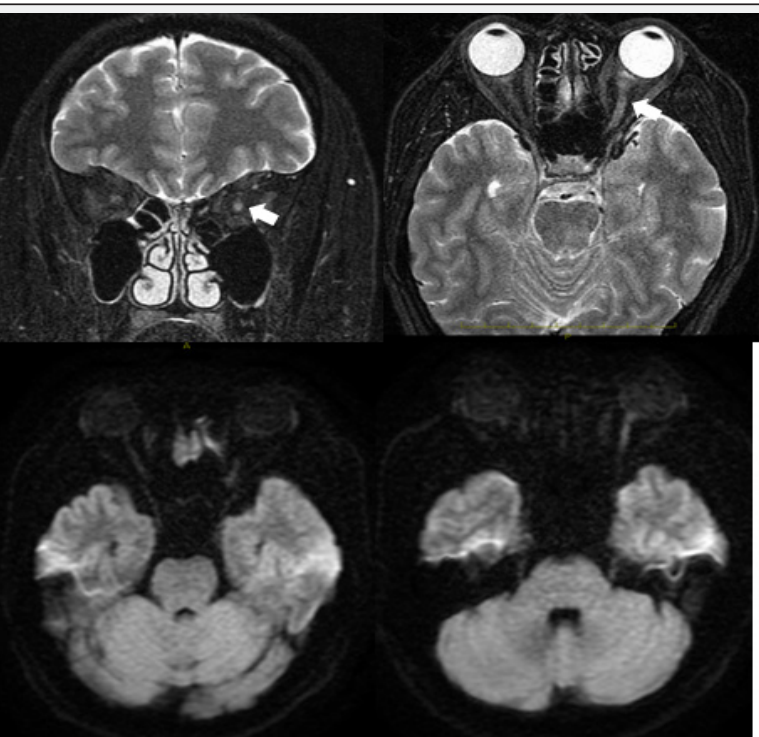

Figure 3: Brain MRI showed hyperintesity of left optic nerve on T2-weightedimage (arrow) and DWI. 


\section{JOJ Ophthalmology}

Upon presentation in our hospital on February 2017, four months after initial presentation, the LE visual acuity had gradually worsened. No pain with eye movement was present, but slight discomfort was noted by the patient. The patient also complained of subjective central visual field defect and color vision abnormalities. By this time, the RE visual acuity was 1.5 but LE decreased to only 0.08 , with positive relative afferent pupillary defect (RAPD) of the left eye and normal pupillary reflex on both eyes. Ocular motility of both eyes was normal. All findings were normal on the RE. Left eye Ishihara plates color vision test was abnormal and perimetry (Goldman and Humphrey 30-2) revealed LE cecocentral scotoma (Figure 4). Fundus examination showed resolution of LE optic disc swelling and a mild thickening of inferior optic disc margin (Figure 5). No optic disc swelling or leakage was found on fluorescein angiography. Ganglion cell analysis showed thinning of macular ganglion cells of the LE (Figure 6). Electrophysiology studies revealed normal flash full-field ERG on both eyes, with slightly reduced focal macular ERG parameters of the LE, severe diminution amplitude with prolonged implicit time on pattern and flash VEP to a point of almost indistinguishable waveform, and reduced photopic negative response (phNR) of the LE.
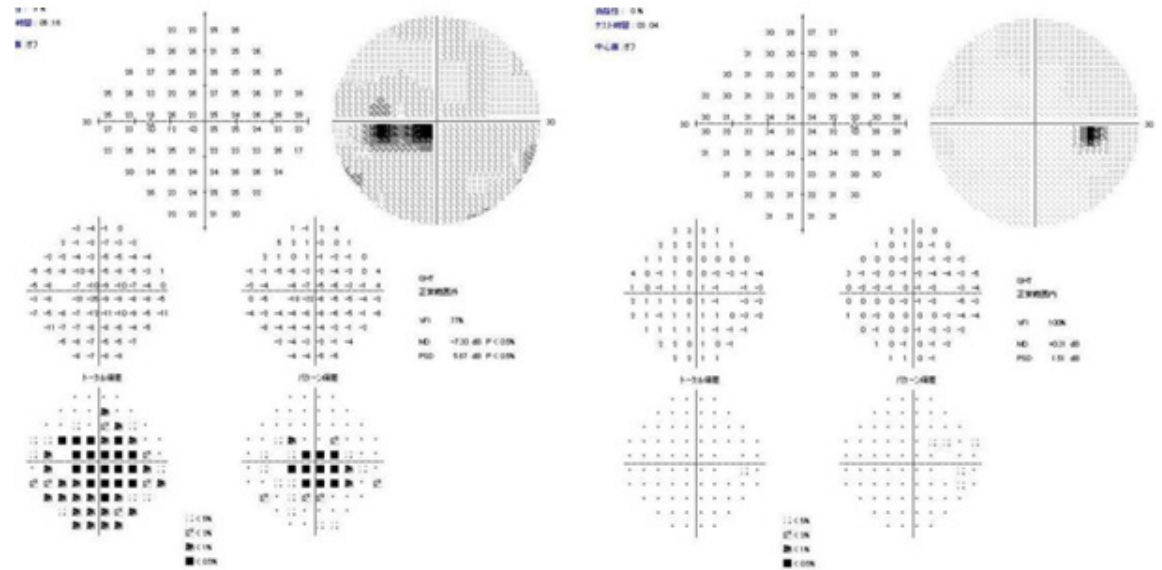

Figure 4: Cecocentral scotoma visual field defect of the LE with otherwise normal RE visual field.
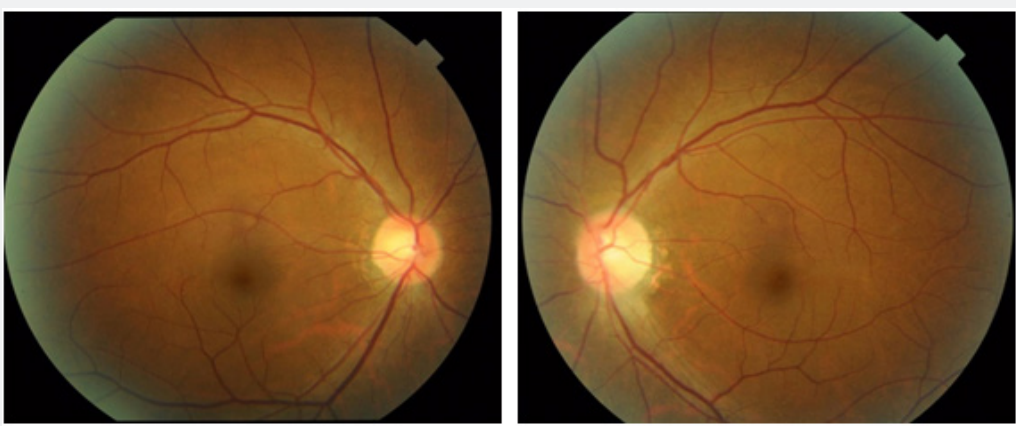

Figure 5: Four months after initial complaint, the LE optic disc showed resolution of optic disc swelling, with a mild inferior nerve fiber layer thickening.
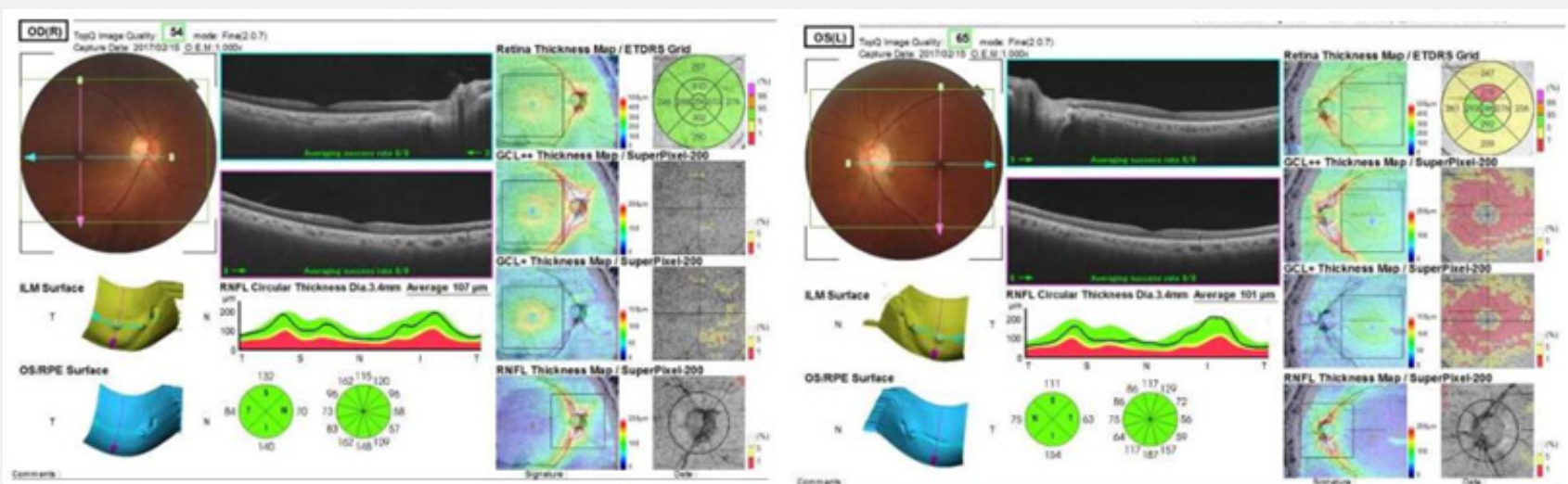

Figure 4: Cecocentral scotoma visual field defect of the LE with otherwise normal RE visual field. 


\section{JOJ Ophthalmology}

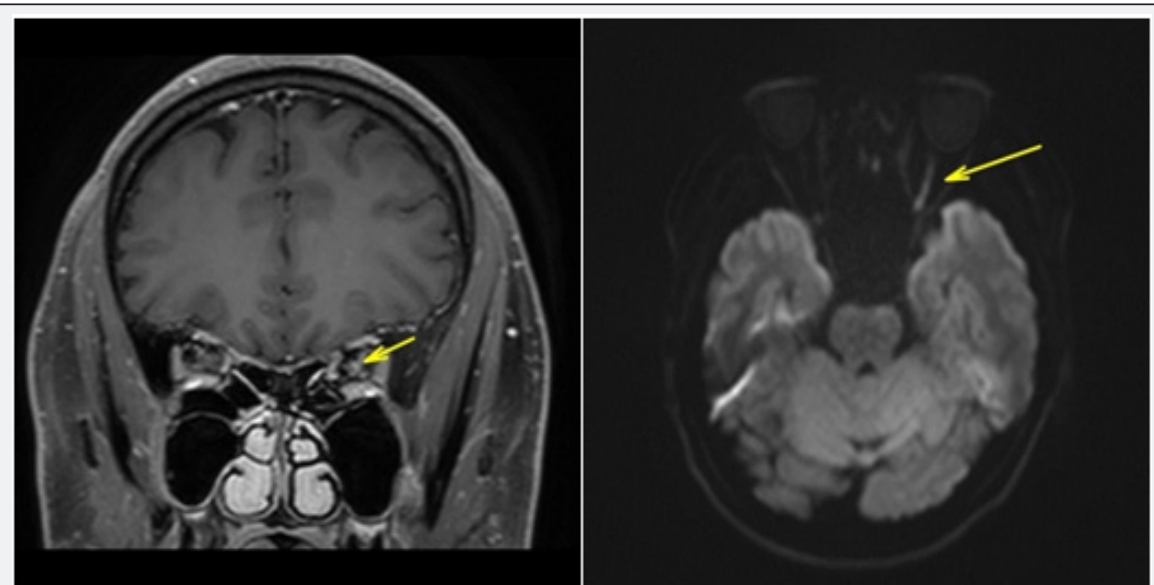

Figure 7: Re-imaging of the brain using MRI four months after initial presentation still showing some enlargement and enhanced optic nerve of the LE on T1-gadolinium enhanced image (left) and DWI (right) (arrow).

MRI brain imaging was re-performed to exclude other etiologies as the cause of visual loss in this patient. No signs of white matter demyelination were present. Four months after the first episode of attack, the LE optic nerve still showed a T2-weighted image and DWI hyper intense optic nerve, with gadolinium enhancement on T1-weighted image (Figure 7).

Routine blood examination wasnormal and further workup found no signs of demyelination, infectious, or autoimmune etiology as the cause of atypical optic neuritis in this patient. Cerebrospinal fluid opening pressure was normal; with normal analysis for cells, glucose and protein content. No CSF oligoclonal band was detected. Due to the atypical findings of this case, genetic testing for specific Leber's hereditary optic neuropathy (LHON) mutation was performed. The result was positive for point mutation for mitochondrial gene 14484 .

\section{Discussion}

Leber's hereditary optic neuropathy remains as the most common cause of optic neuropathy due to primary mutation of mtDNA, with prevalence ranging from one in 31,000 to 526,000 throughout different regions of the world [1]. The symptomatic phase usually develops between the age of 15 to 40 years. The specific mitochondrial point mutations in mt11778, mt14484, and $\mathrm{mt} 3460$ creates disturbance of the enzyme coding needed for adenosine triphosphate production in the electron transport oxidative phosphorylation pathway, resulting in the interruption of the energy chain and triggering oxidative stress [2]. This dysfunctional energy pathway then causes selective retinal ganglion cells (RGC) apoptosis, which are particularly sensitive to mitochondrial dysfunction and metabolic insult [1,3]. Most LHON cases in Japan bear the 11778 mtDNA point mutation $[3,16]$. Our patient was found with the less frequent mutation of 14484 mtDNA.

This case presented with several atypical clinical features of LHON: unilateral involvement, positive RAPD, swollen optic disc with leakage of fluorescein angiography, and evidence of optic nerve hyperintensity with enhancement on neuroimaging.
Such atypical features during initial presentation might give an impression of other optic neuropathies as the etiology for visual loss in this case. The atypical clinical features of LHON often.

The first interesting discussion point of this case report would be regarding the findings on MRI. Leber's hereditary optic neuropathy (LHON) usually demonstrates normal findings on MRI [7]. Few studies, however, reported hyperintensity and enhancement of the visual pathway involved in LHON [5,813]. This case presented with atypical features of LHON, with persistent optic nerve hyperintensity and enhancement of the involved optic nerve shown in both the acute phase, and 4 months later during the chronic phase.

Enhancement of optic nerve during acute phase of LHON is rarely seen, although a handful of cases had been reported [10,12,13,17-19]. Earlier reports demonstrated optic nerve hyperintensity in T1-weighted image and Short Time Inversion Recovery (STIR) image [8,9]. Other reported cases with visual pathway hyperintensity and enlargement on T2-weighted MRI, during acute phase of LHON, and persisted throughout the chronic phase of the disease, with or without contrast enhancement [8-10,12,13,17-19]. The reported location of hyperintensity lesion includes the optic nerve, optic chiasm and along the pre-geniculate visual pathway of optic tract [8$10,12,13]$. Optic nerve sheath distention had also been reported in one patient with LHON, demonstrate using orbital ultrasound, computed tomography and MRI [20]. All reported cases have mt11778 DNA mutation.

The factors influencing signal intensity on MRI includes proton density and their immediate physico-chemical environment, thus giving us information regarding the fluid and cellular environment of the axons [9]. It is postulated that the abnormal optic nerve MRI signal in LHON might be due to edema and axonal swelling in the acute phase and gliosis along with axonal degeneration in the more chronic phase of LHON $[9,15,21]$. The presence of enhancement suggested a disruption of blood-brain barrier, causing an inflammatory-like mechanism 
as the underlying pathophysiology [12]. This might explain the MRI findings in our patient.

Bargiegla et al. [22] suggest an overlapping course of LHON and multiple sclerosis, as a complex of neuroinflammation process. First described in 1926 by Mauksch et al. the term LHON-MS was established to describe the association between CNS inflammatory demyelination and LHON [22]. Abnormalities found in brain MRI of LHON patients often demonstrated MS-like changes of white matter, especially in female 11778 mtDNA mutation patients [15]. However, our patient did not demonstrate any white matter demyelination in neuroimaging or any neurological symptoms typical for MS, therefore the LHON-MS etiology is unlikely to be the cause of optic nerve hyperintensity and enhancement.

The natural course of LHON includes severe loss of retinal ganglion cells and axons, especially in acute phase as demonstrated by structural imaging using OCT and electrophysiology test for ganglion cells (pattern visual evoked potential, pattern electroretinography, and photopic negative response), followed by secondary changes including demyelination and gliosis [23]. Post mortem visual pathway histopathological specimen in patients with classical clinical profile of LHON demonstrated structural changes of severe retinal ganglion cell and axon loss, central demyelination of optic nerves, chiasm, and optic tracts with evidence of gliosis [24]. These structural changes might explain the T2 hyperintense signal on neuroimaging, however, given the relatively few number of autopsy reports in LHON patient, it remains undetermined. Surprisingly, visual pathway abnormalities of LHON are rarely detected on MRI. However, the recent increasing number of hyperintensity of the visual pathway, from the optic nerve all the way to the pre-geniculate pathway might suggest that maybe we might have not been paying attention to visual pathway neuroimaging abnormalities in LHON. Low imaging quality and lack of specific view of the visual pathway had also been proposed as the cause of the low number of visual pathway bright signal on MRI [10].

The second point of discussion would be the possibility of coincidental optic neuritis attack in a LHON mutation positive patient in this case. However, it should be kept in mind that LHON can be mistakenly diagnosed as optic neuritis, especially earlier during its course before bilateral involvement occurs. RiordanEva et al. [15] reported out of 94 individuals with confirmed LHON-specific mtDNA mutation, $24 \%$ experienced pain around the affected eye or with eye movement, and 4 patients noted Uthoff's phenomenon; with $45 \%$ of female with 11778 mutation had a multiple sclerosis -like illness. Visual loss in LHON typically affects both eyes, more often simultaneously than sequentially. Therefore, LHON could easily be misdiagnosed as optic neuritis and poor response to steroid pulse therapy should alarmed the possibility of LHON as the cause of visual loss [14]. LHON could be difficult to differentiate from optic neuritis, especially in East Asia population where bilateral optic papillitis is more common than in the western counterpart $[14,25]$. However, this case presented with unilateral optic nerve involvement. The fellow eye involvement typical of LHON was undetected in this case, due to the failure of routine follow-up in this patient, although unilateral involvement had been reported in 11778 mutation group, 4 years after the onset of initial visual loss [15].

The diagnosis of LHON is confirmed by the presence of LHON specific mutation, with classic symptoms and fundus findings. Typical optic disc and vascular changes findings include pseudo-edematous disc, teleangiectatic vessels, and thickening of retinal nerve fiber layer, with no leakage on fluorescein angiography, differentiating them from other inflammatory optic neuropathies. These changes have also been noted in asymptomatic patients with no visual symptoms $[4,5]$. The classic visual field defect is central or cecocentral scotoma, with abnormal color vision. Diminution of visual evoked potential wave is present suggesting optic nerve dysfunction. Differentiation of optic disc pseudoedema in LHON from other true disc edema in other optic nerve diseases includes the use of fluorescein angiography; in which no disc leakage is observed in pseudoedema of LHON $[1,15]$. No abnormality is usually found in optic nerve or brain MRI [7]. This case, however, presented with unilateral optic nerve swelling, optic disc leakage on late FA phase and optic nerve hyperintensity with contrast enhancement on MRI; an atypical features of LHON signifying an endothelial dysfunction of the optic nerve vessels and raising suspicion of inflammatory etiology. Due to these atypical findings, the diagnosis of optic neuritis was highly suspected on the initial presentation [15]. There are few reports of optic disc leakage during the acute phase of LHON. One case of fluorescein leakage was due to congenitally anomalous optic disc, while the other was mistakenly diagnosed with optic neuritis until DNA analysis was performed [14,15]. Sugisaka et al. [26] reported presence of circumpapillary microangiopathy of the optic disc might be useful to differentiate LHON from optic neuritis. However, we did not have such finding in our patient. Therefore, due to similarity of clinical presentation, it would be difficult to differentiate the two etiologies without LHON-specific DNA mutation analysis. Diagnosis confirmation is crucial, especially in LHON because of the clinical course, prognosis and hereditary pattern of the disease. Adequate genetic counseling is needed in case of LHON. Visual prognosis in LHON is poor, with most affected will remain blind, although spontaneous visual improvement is possible to some degree, most likely within a year and frequently associated with 14484 mutation [27].

The final interesting point to discuss in this case report was whether the onset of LHON-related visual loss in this case could have been triggered by presence of insult to the optic nerve due to other etiologies, e.g optic neuritis in this case. Symptomatic phase of LHON had been reported to be elicited not only by exposures to certain agents such as smoking, alcohol, certain medication, but also by optic nerve insults due 
to other optic neuropathies or even trauma $[10,15,28]$. Lamirel et al. [10] reported one case of 11778 mtDNA LHON-specific mutations with confirmed diagnosis of idiopathic intracranial hypertension with papilledema, and later in the course of the disease experience acute visual loss related to LHON, raising a question of papilledema-induced LHON-related visual loss. It was hypothesized that papilledema in IIH poses further disturbance to pre-existing sub-normal axoplasmic transport in LHON, possibly responsible for additional imbalance of oxidative regulation in retinal ganglion cells. However, to our knowledge no further studies had been made to ascertain the correlation between optic nerve insults and onset of visual loss in LHON.

The diagnosis of LHON should be suspected in atypical presentation of optic neuritis, especially with chronic abnormal bright T2 findings on MRI months after initial visual onset. The absence of classical typical clinical features of LHON often leads to initial misdiagnosis in the absence of specific mtDNA mutation identification $[14,15,18]$.

\section{References}

1. Meyerson C, Van Stavern G, McClelland C (2015) Leber hereditary optic neuropathy: Current perspectives. Clin Ophthalmol 9: 1165-1176.

2. Burde R, Savino P, Trobe J (2002) Optic neuropathies. In: Burde RM, et al. (Eds.), Clinical decisions in neuro-ophthalmology. Mosby, Missouri, p. 27-53.

3. Ueda K, Morizane Y, Shiraga F, Shikishima K, Ishikawa $H$, et al. (2017) Nationwide epidemiological survey of leber hereditary optic neuropathy in japan. J Epidemiol 27(9): 447-450.

4. Piotrowska A, Korwin M, Bartnik E, Tonska K (2015) Leber hereditary optic neuropathy - historical report in comparison with the current knowledge. Gene 555(1): 41-49.

5. Vaphiades MS, Newman NJ (1999) Optic nerve enhancement on orbital magnetic resonance imaging in leber's hereditary optic neuropathy. J Neuroophthalmol 19(4): 238-239.

6. Yu-Wai-Man P, Turnbull DM, Chinnery PF (2002) Leber hereditary optic neuropathy. Journal of medical genetics 39: 162-169.

7. Newman NJ (1993) Leber's hereditary optic neuropathy: New genetic considerations. Arch Neurol 50(5): 540-548.

8. Dotti MT, Caputo N, Signorini E, Federico A (1992) Magnetic resonance imaging findings in leber's hereditary optic neuropathy. Eur Neurol 32(1): 17-19.

9. Kermode AG, Moseley IF, Kendall BE, Miller DH, MacManus DG, et al. (1989) Magnetic resonance imaging in leber's optic neuropathy. J Neurol Neurosurg Psychiatry 52(5): 671-674.

10. Lamirel C, Cassereau J, Cochereau I, Vignal-Clermont C, Pajot O, et al. (2010) Papilloedema and MRI enhancement of the prechiasmal optic nerve at the acute stage of leber hereditary optic neuropathy. J Neurol Neurosurg Psychiatry 81(5): 578-580.

11. Beck RW, Trobe JD, Moke PS, Gal RL, Xing D, et al. (2003) High- and low-risk profiles for the development of multiple sclerosis within 10 years after optic neuritis: Experience of the optic neuritis treatment trial. Arch Ophthalmol 121(7): 944-949.

12. Ong E, Biotti D, Abouaf L, Louis-Tisserand G, Tilikete C, et al. (2013) Teaching neuroimages: Chiasmal enlargement and enhancement in leber hereditary optic neuropathy. Neurology 81(17): e126-e127.

13. van Westen D, Hammar B, Bynke G (2011) Magnetic resonance findings in the pregeniculate visual pathways in leber hereditary optic neuropathy. J Neuroophthalmol 31(1): 48-51.

14. Hsu TK, Wang AG, Yen MY, Liu JH (2014) Leber's hereditary optic neuropathy masquerading as optic neuritis with spontaneous visual recovery. Clin Exp Optom 97(1): 84-86.

15. Riordan-Eva P, Sanders MD, Govan GG, Sweeney MG, Da Costa J, et al. (1995) The clinical features of leber's hereditary optic neuropathy defined by the presence of a pathogenic mitochondrial DNA mutation. Brain 118 ( Pt 2): 319-337.

16. Mashima Y, Yamada K, Wakakura M, Kigasawa K, Kudoh J, et al. (1998) Spectrum of pathogenic mitochondrial DNA mutations and clinical features in japanese families with leber's hereditary optic neuropathy. Curr Eye Res 17(4): 403-408.

17. Batioglu F, Atilla H, Eryilmaz T (2003) Chiasmal high signal on magnetic resonance imaging in the atrophic phase of leber hereditary optic neuropathy. J Neuroophthalmol 23(1): 28-30.

18. Honda H, Tsujihata M, Ochi M, Satoh A, Tomita I, et al. (2006) [Hyperintense optic nerve lesion on t2-weighted MRI imaging in the acute stage of leber's hereditary optic neuropathy: A case report]. Rinsho Shinkeigaku 46(4): 294-296.

19. Phillips PH, Vaphiades M, Glasier CM, Gray LG, Lee AG (2003) Chiasmal enlargement and optic nerve enhancement on magnetic resonance imaging in leber hereditary optic neuropathy. Arch Ophthalmol 121(4): 577-579.

20. Smith JL, Tse DT, Byrne SF, Johns DR, Stone EM (1990) Optic nerve sheath distention in leber's optic neuropathy and the significance of the "wallace mutation". J Clin Neuroophthalmol 10(4): 231-238.

21. Newman NJ (2011) Leber hereditary optic neuropathy: Some new observations. J Neuroophthalmol 31(1): 3-5.

22. Bargiela D, Chinnery PF (2017) Mitochondria in neuroinflammation multiple sclerosis (ms), leber hereditary optic neuropathy (lhon) and lhon-ms. Neurosci Lett.

23. Majander A, Robson A, João C, Holder G, Chinnery P, et al. (2017) The pattern of retinal ganglion cell dysfunction in leber hereditary optic neuropathy. Mitochondrion 36: 138-149.

24. Adams JH, Blackwood W, Wilson J (1966) Further clinical and pathological observations on leber's optic atrophy. Brain 89(1): 15-26.

25. Zhang YJ, Du Y, Li K, He JF (2014) Re: Leber's hereditary optic neuropathy masquerading as optic neuritis with spontaneous visual recovery. Clin Exp Optom 97(2): 187.

26. Sugisaka E, Ohde H, Shinoda K, Mashima Y (2007) Woman with atypical unilateral leber's hereditary optic neuropathy with visual improvement. Clin Exp Ophthalmol 35(9): 868-870.

27. Yu-Wai-Man P, Votruba M, Moore AT, Chinnery PF (2014) Treatment strategies for inherited optic neuropathies: Past, present and future. Eye 28(5): 521-537.

28. Apinyawasisuk S, Chan JW, Arnold AC (2016) Trauma-associated leber hereditary optic neuropathy. Neuro-ophthalmology 40(4): 192-196. 


Your next submission with Juniper Publishers
will reach you the below assets
- Quality Editorial service
- Swift Peer Review
- Reprints availability
- E-prints Service
- Manuscript Podcast for convenient understanding
- Global attainment for your research
- Manuscript accessibility in different formats
( Pdf, E-pub, Full Text, Audio)
- Unceasing customer service
Track the below URL for one-step submission
https://juniperpublishers.com/online-submission.php

